\title{
Optical and Wireless Convergence
}

\author{
Wansu Lim, Milos Milosavljevic, Pandelis Kourtessis, John M. Senior \\ Optical Networks Group, Science and Technology Research Institute (STRI), University of Hertfordshire, Hatfield, AL10 9AB, UK \\ E-mail:m.milosavljevic@herts.ac.uk,Tel: +441707286049
}

\begin{abstract}
This paper presents the requirements and possible solutions for wireless convergence in next generation PONs. System level simulation of LTE was performed to evaluate two different approaches for connecting eNBs to ONUs.

(C2010 Optical Society of America
\end{abstract}

OCIS codes: 060.4250 (Networks), 060.4510 (Optical communications)

\section{Introduction}

With developments in broadband wireless networks, driven primarily by long term evolution (LTE), data rates in the range of hundreds of Mbps are expected to be supported over next generation radio cells. This will unavoidably lead to a higher density of base station deployment. High bandwidth, cost-effective feeder links will be required as a result to connect each of these base stations to a common main central office. This can be achieved with traditional IP backhauling requiring QoS mapping between the eNB and ONUs in order to optimise the performance of both the optical and wireless networks. Therefore, this paper presents requirements and possible solutions for the optical and wireless convergence in order to efficiently support $4 \mathrm{G}$ and beyond mobile networks.

\section{Next generation wireless network backhauling}

The mobile infrastructure LTE equipment market is forecast to grow at a compound annual growth rate (CAGR) of $81 \%$ over the next 5 years, and reach $\$ 8$ billion by 2015 [1]. More specifically, with respect to the Evolved Packet Core (EPC), equipment supporting these $4 \mathrm{G}$ networks is now $5 \%$ of the overall packet core market. However, the EPC segment is expected to grow over $200 \%$ only in 2012 [1]. In addition, the market for the backhaul part of the network is expected to significantly increase over the next few years due to a high demand for bandwidth in existing networks and a high push for high speed Internet access in remote areas [1]. This is particularly relevant for LTE/LTE-Advance deployments, where capacity demands are expected to be one order of magnitude above the current ones. This together with advances in mobile devices would unavoidably lead to very high mobile data traffic across cells. Therefore, to minimise the investment and maintenance costs, a good balance of high speed backhaul transport (i.e., optical networks) with easy to set up and operate wireless networks will be required [2].

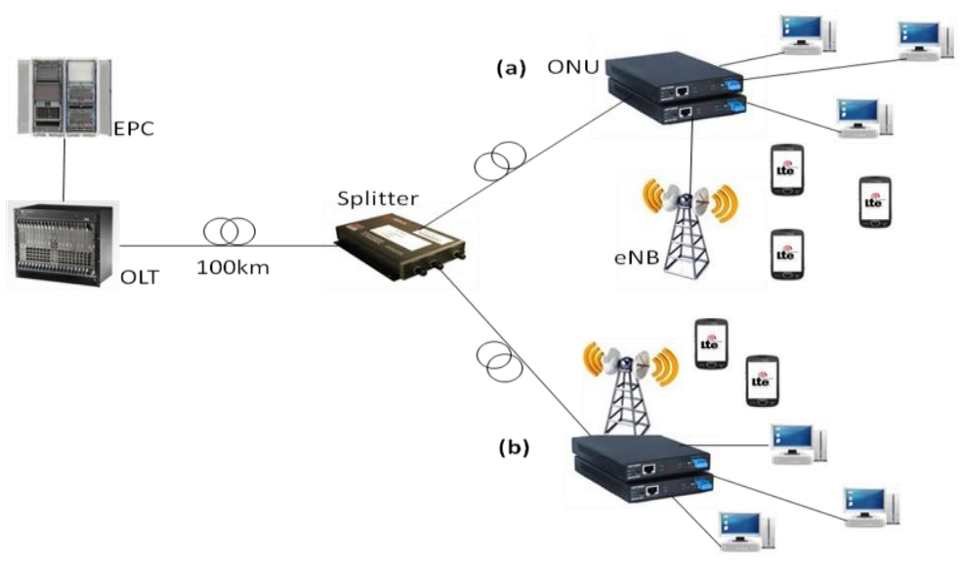

Fig. 1. PON and LTE convergence: (a) independent and (b) hybrid base station operation

The Fig. 1 describes possible options for backhauling LTE/LTE-A over long reach next generation passive optical networks (NG-PONs) [3]. The LTE enhance NodeB (eNB) could be connected directly to an optical network unit (ONU) via an Ethernet port (option (a)) or being integrated within the ONU (option (b)). A hybrid 
solution offers several advantages compared to the independent one since the quality of service (QoS) could be adapted taking into accounts both optical and wireless users. Such systems require an efficient QoS mapping mechanism between optical and wireless queues. With the independent approach the optical scheduler at the optical line terminal (OLT) is unaware of the bandwidth allocation across the wireless cells degrading the QoS.

\section{Network modeling of hybrid and independent converged approach}

To evaluate both approaches a system level simulation test-bed was developed with OPNET, modelling NG-PON2 network based on OFDMA [3] and typical LTE network with inter-side distance of $3 \mathrm{~km}$. For the optical part the number of OFDMA subcarriers was 64 with total number of ONUs of 32. The investigation was based in upstream (similar concept would also apply for downstream) from an eNB to the EPC using novel protocols developed for the OFDMA PONs [4]. In addition, distance between ONU and OLT is $20 \mathrm{~km}$ and total data rate is $10 \mathrm{Gbps}$ [3].
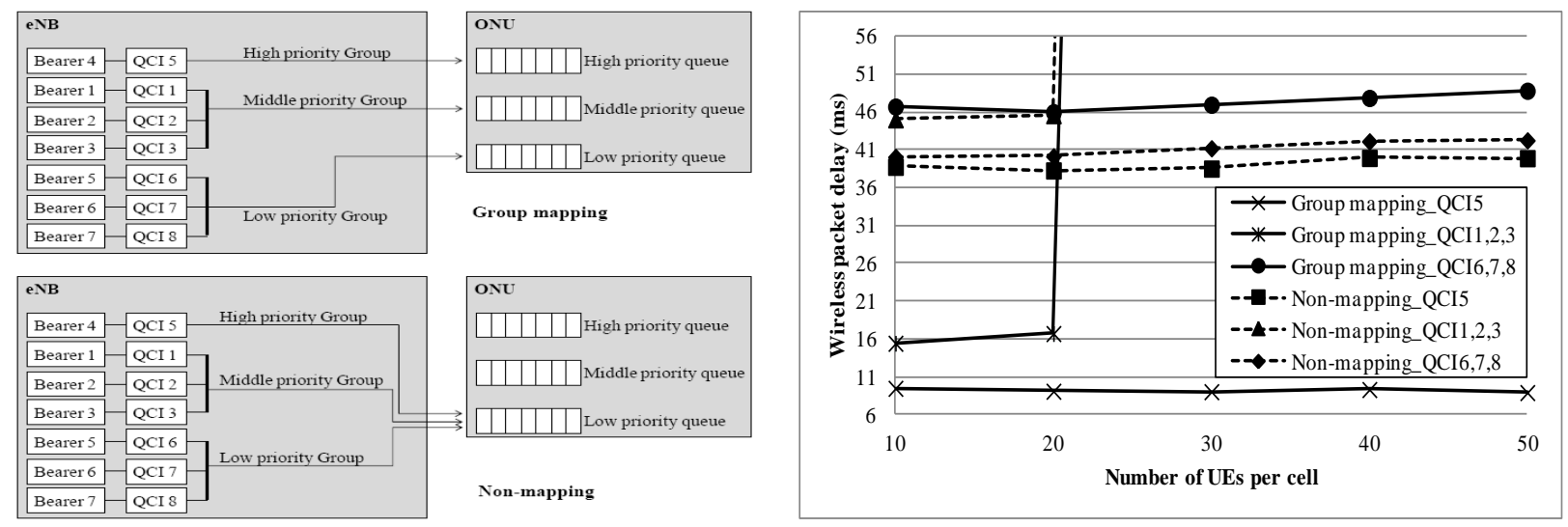

Fig. 2. Packet delay (right) for group mapping (option (b)) versus non-mapping (option (a))

Fig. 2 (right) presents group mapping, contrasted again to non-mapping, implemented by the transition of the 7 LTE bearers to the low priority ONU queue. The QoS channel identifier 5 (QCI5) bearer emulates high priority, while QCI1, 2 and 3 and QCI6, 7 and 8 bearer groups signify respectively, middle and low priorities. The performance of the middle priority group (QCI1, 2 and 3) saturates early at 30 UEs. This can be pinned down to the use of guaranteed bit rate (GBR) services, comprising, in the 7 bearers mapping, the middle priority group. The packet delay of the low priority group is $6 \mathrm{~ms}$ higher with group mapping (hybrid scenario of Fig. 1), compared to non-mapping (independent approach), because of the strict priority followed with intra scheduling inside ONUs.

\section{Conclusions}

With the ever growing mobile data traffic, efficient backhauling transport links (based on PON infrastructure) are required. To that extent, two IP backhauling approaches are presented to connect eNBs to ONUs. The results obtained in terms of QCI delay with respect to number of users per cell suggest that the hybrid approach as opposed to independent approach provides comprehensive QoS mapping between the ONU and eNB queues.

\section{Acknowledgments}

This work was supported by the ACCORDANCE project, through the 7th ICT Framework Programme.

\section{References}

[1] Cisco Visual Networking Index: Global mobile Data Traffic Forecast. [Online], Available at: www.cisco.com

[2] M. Milosavljevic, M. P. Thakur, P. Kourtessis, J. E. Mitchell, and J. M. Senior, "Demonstration of wireless backhauling over long-reach PON,” IEEE/OSA Journal of Lightwave Technology, vol. 30, no. 5, pp. 811 - 817, Mar. 2012.

[3] EU FP7 ACCORDANCE project [Online], Available at: http://www.ict-accordance.eu/

[4] Wansu Lim, Pandelis Kourtessis, Milos Milosavljevic, John M. Senior, "Dynamic Subcarrier Allocation for OFDMA-PONs with Monitoring Mechanism," ANIC 2011, Toronto, Canada, June 2011 